\title{
Chemistry and Mechanism of One-Step Formation of Graphene from Agrowaste
}

\author{
Jhuma Debbarma ${ }^{1}$, Peetam Mandal ${ }^{1}$, Mitali Saha ${ }^{1, *(D)}$ \\ 1 Department of Chemistry, National Institute of Technology Agartala, 799046, Tripura, India \\ * Correspondence: mitalichem71@gmail.com;
}

Scopus Author ID 55779373600

Received: 13.06.2020; Revised: 4.07.2020; Accepted: 4.07.2020; Published: 7.07.2020

\begin{abstract}
The one-step synthesis of high-quality graphene derivatives via CVD process has gained considerable importance nowadays for high-performance electronics and sensors. However, the use of harsh chemicals, high temperature, sensitivity, and the problem of separation of graphene from the substrate, motivated the one-step synthesis of graphene from a non-graphitic precursor, bypassing the use of graphite. In this paper, we have reported for the first time, the synthesis of graphene nanosheets from sugarcane bagasse at the normal atmospheric condition in a single step, avoiding the formation of GO. Here, the pyrolysis of sugarcane bagasse was carried out in the temperature range of $250-450^{\circ} \mathrm{C}$ in the presence of sodium hydroxide. The results suggested that even the low temperature $\left(250-450^{\circ}\right.$ C) facilitated the development of graphitic planes via condensation and aromatization of the glucose monomers present in the precursor. The XRD pattern showed $2 \theta$ at around $25^{\circ}$ in each case, which confirmed the formation of graphene instead of GO. The FESEM, TEM, and EDX analysis proved the formation of few-layer nanosheets of graphene from carbon-rich waste precursors in a single step.
\end{abstract}

Keywords: Sugarcane bagasse; $\mathrm{NaOH}$; pyrolysis; few layer graphene.

(C) 2020 by the authors. This article is an open-access article distributed under the terms and conditions of the Creative Commons Attribution (CC BY) license (https://creativecommons.org/licenses/by/4.0/).

\section{Introduction}

During the last decade, graphene oxide (GO) and reduced graphene oxide (r-GO) were reported from graphite powders involving a two-step process [1-3]. Simultaneously, a new strategy to prepare GO and r-GO was also adopted using non-graphitic biomass and agro-waste materials, avoiding the graphite precursor [4-10]. Very recently, the direct synthesis of graphene derivatives has gained considerable importance, bypassing the formation of GO [11]. Despite several shortcomings such as specialized equipment, harsh chemicals, a large amount of energy requirements, etc. numerous reports were available on CVD as the most researched method of mass production of graphene especially, for high-tech electronics and sensors applications [12-18]. However, its sensitivity towards the change of parameters and the separation of graphene from the substrate without affecting the properties of the material or damaging the structure of the graphene is highly challenging. In addition, the commercial and economical production of graphene may prevent this method from becoming the preferred method of synthesis of graphene in the future.

In view of these observations and in continuation of our research work on a two-step synthesis of graphene, a one-step process has been reported for the first time in the synthesis of graphene from a non-graphitic agro-waste, which has been untouched till now [19]. Here, the pyrolysis of sugarcane bagasse was carried out at three different temperatures viz. $250^{\circ} \mathrm{C}$, 
$350^{\circ} \mathrm{C}$, and $450^{\circ} \mathrm{C}$ in the presence of sodium hydroxide. The effect of temperature and time was studied for the formation of graphene, and the mechanism has been explained. The results suggested that the in-situ use of sodium hydroxide hindered the attack of oxygen during pyrolysis. The mechanism confirmed the participation of glucose monomers in the aromatization and condensation process, leading to the formation of nanosheets of graphene in a single step.

\section{Materials and Methods}

Sugarcane bagasse was collected from a local market. P-XRD pattern was recorded by Bruker D8 advance diffractometer in the $2 \theta$ range of 5-60 . FE-SEM, along with EDX images, were observed by using Sigma-300, Carl Zeiss, HRTEM images were collected using a Jeol JEM-2100 microscope at an acceleration voltage of $200 \mathrm{kV}$.

Bagasse sample was cut into small pieces, washed thoroughly with deionized water several times for removing dust, dried in sunlight for 2 to 3 days, and finally dried in the oven for 24 hours. The finely grinded powder after drying was mixed with sodium hydroxide in a 1:1 ratio and subjected to pyrolysis at different temperatures viz. $250^{\circ} \mathrm{C}, 350^{\circ} \mathrm{C}$, and $450^{\circ} \mathrm{C}$. The obtained shiny black colored powder products were washed with $2 \mathrm{M}$ nitric acid and deionized water thoroughly for removing the unwanted products, then filtered and dried overnight.

\section{Results and Discussion}

In recent years, graphene and its derivatives have been gained considerable attention towards glucose-sensing due to high surface area, which can serve as a superior platform for fabricating new composites in glucose sensing[20-30]. The main aim was to prepare the graphene from non-graphitic agro-waste material in a single step instead of functionalized graphene (GO) without using any inert atmosphere. Moreover, the purpose of using $\mathrm{NaOH}$ was just to prevent the formation of GO. The strong reducing nature of $\mathrm{NaOH}$, which might have resulted in graphene during a long heating time, due to the absence of an inert atmosphere. Hence, when the 1:1 ratio of sugarcane bagasse and sodium hydroxide was pyrolyzed at various temperatures, black colored crystalline products were obtained in each case. In the XRD pattern (Figure 1), it was observed that when the sugarcane bagasse was pyrolyzed at $250^{\circ} \mathrm{C}$ for $2 \mathrm{hrs}$, a peak was observed at $2 \theta$ of $27.4^{\circ}$. In the case of heating at $350^{\circ} \mathrm{C}$ for $2 \mathrm{hrs}$, a more intense peak was obtained (Figure $1 \mathrm{~b}$ ) at $2 \theta$ of $25.6^{\circ}$. Figure 1c showed a significant decrease in the peak intensity at around $2 \theta$ of $24.9^{\circ}$. The results showed that on heating at $250^{\circ} \mathrm{C}$ for $2 \mathrm{hrs}$, both graphene nanosheets and nanoparticles were formed, indicating that the glucose monomers were not getting sufficient time for aromatization and condensation. The heattreatment at $450^{\circ} \mathrm{C}$ for $1 \mathrm{hr}$, although resulted in the formation of nanosheets, was found to be broken due to high temperature. In contrast to $250^{\circ} \mathrm{C}$ and $450^{\circ} \mathrm{C}$, the pyrolysis at $350^{\circ} \mathrm{C}$ for 2 hrs resulted in the formation of graphene nanosheets only, which confirmed that this temperature and the retention time was essentially required for the condensation and aromatization of glucose monomers and finally to the development of graphitic planes. Moreover, the formation of 002 planes in all the temperatures confirmed that sodium hydroxide prevented the formation of GO at normal atmospheric conditions during pyrolysis.

The SEM images showed the formation of nanosheets (Figures 2a, 3a, b, and 4a) at all three temperatures. The elemental composition of the energy-dispersive X-ray spectroscopy 
(EDX) analysis exhibited the signals of carbon and oxygen (Figure 2b, 3c, and 4b) and confirmed that sodium hydroxide successfully prevented the formation of GO.

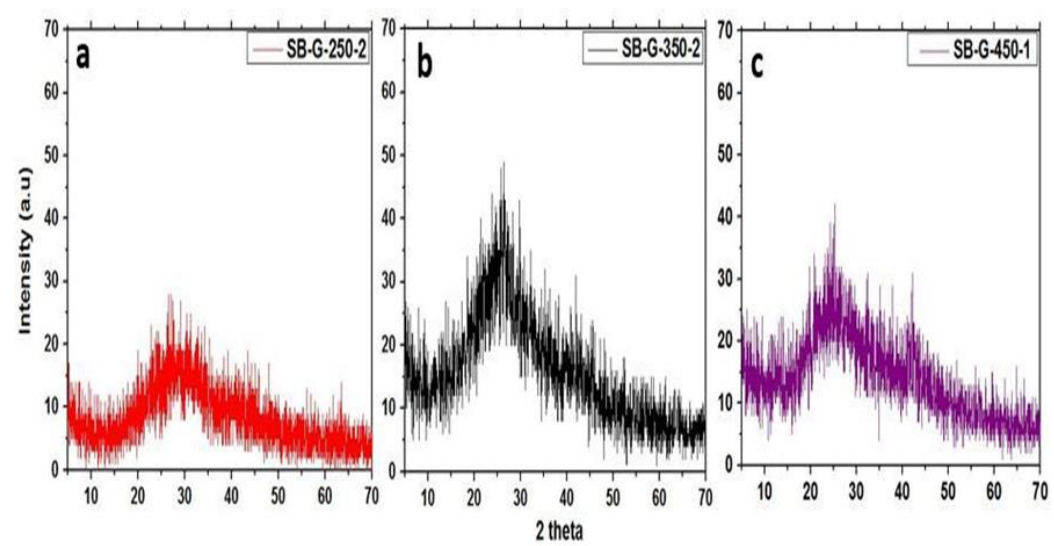

Figure 1. XRD patterns of graphene at various temperatures viz. $250^{\circ} \mathrm{C}(\mathrm{a}), 350^{\circ} \mathrm{C}(\mathrm{b})$ and $450^{\circ} \mathrm{C}(\mathbf{c})$.

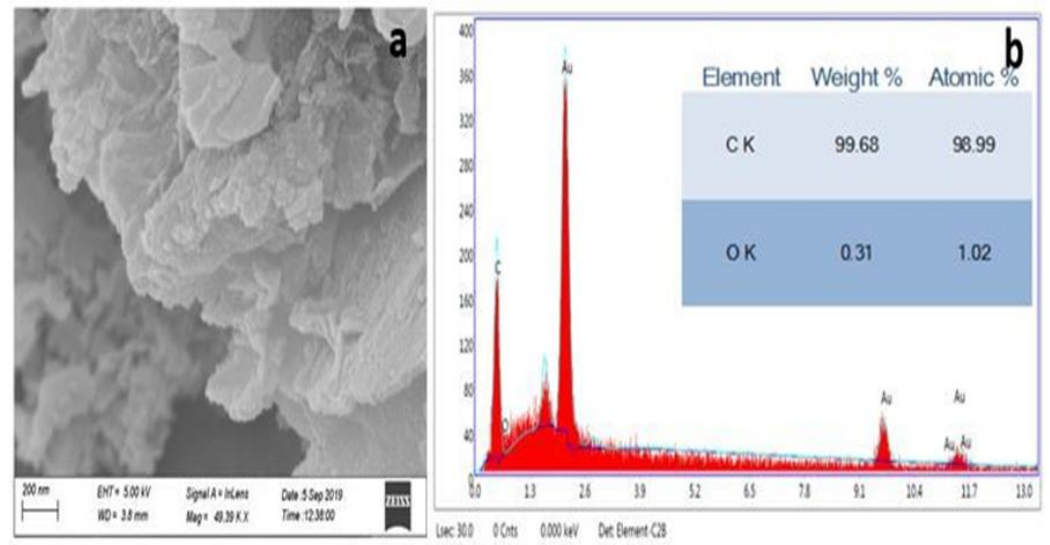

Figure 2. SEM image (a) and EDX of graphene (b) at $250^{\circ} \mathrm{C}$ temperature.

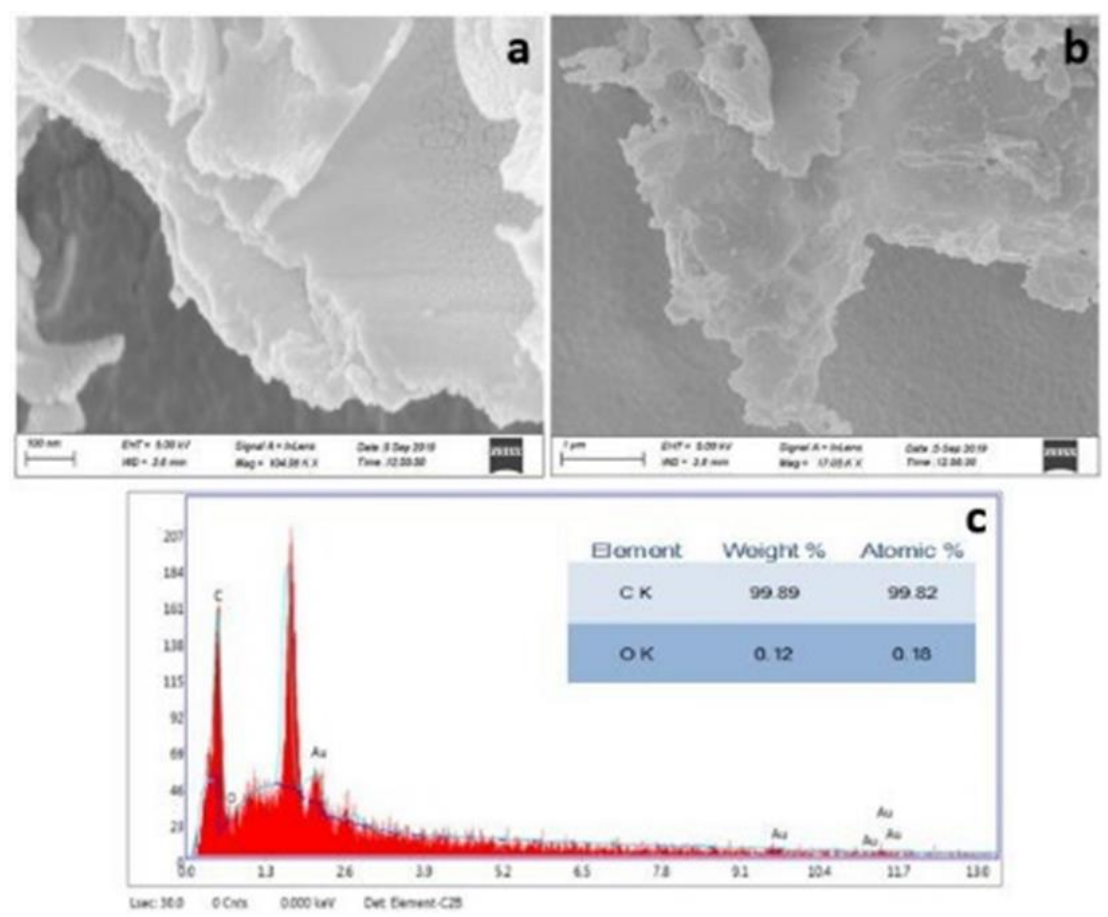

Figure 3. SEM images (a), (b) and EDX of graphene (c) at $350^{\circ} \mathrm{C}$ temperature. 


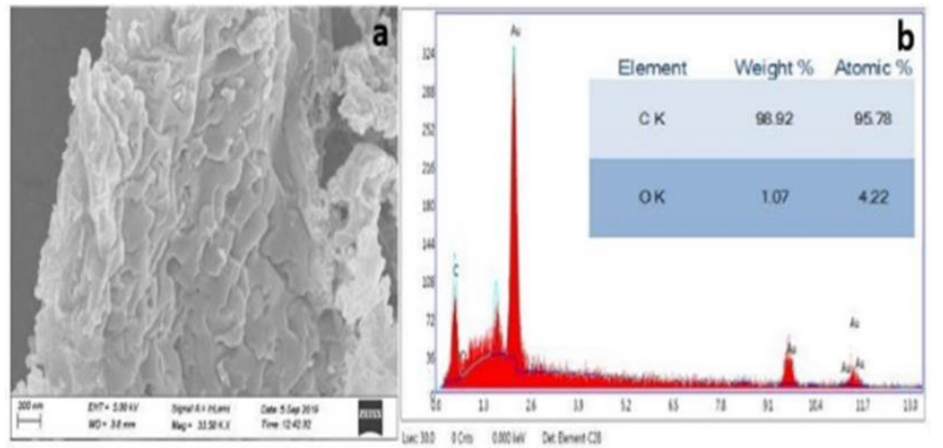

Figure 4. SEM images (a) and EDX of graphene (b) at $450^{\circ} \mathrm{C}$ temperature.

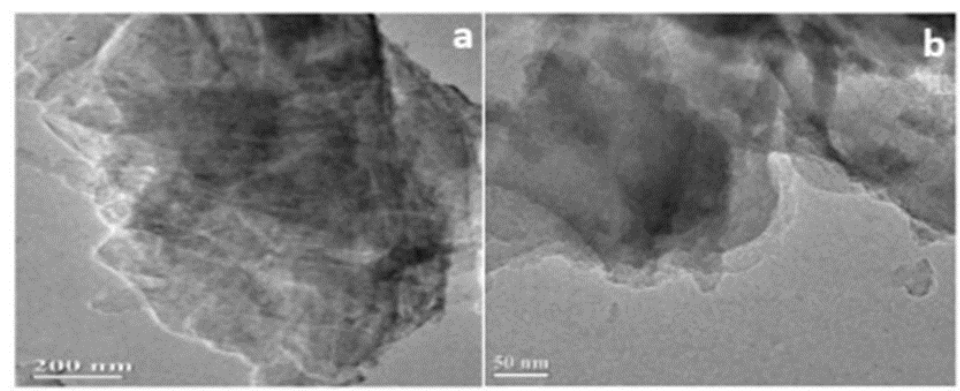

Figure 5. TEM images (a) and (b) at $350^{\circ} \mathrm{C}$ temperature.

The mechanism has been presented in Figure 6, showing the chemistry behind the formation of graphene nanosheets. The carbon-rich bagasse primarily contains cellulose, which contains glycosidic bond and hydroxyl groups. When bagasse was pyrolyzed at the range of $250-450^{\circ} \mathrm{C}$, which led to the degradation of glucose monomers and these monomers, contain aldehydic and hydroxyl groups. It is well known that the formation of a cyclic hemiacetal structure can be possible when the hydroxyl group at the fifth carbon of glucose gets attached to the aldehydic group of the first carbon [31]. Since the resulting cyclic structure from glucose monomers resembles pyran, having six-membered heterocyclic rings, it might be possible that during heating at the range of $250-450^{\circ} \mathrm{C}$, a large number of glucose monomers joined together by glycosidic bonds. Further, the condensation and aromatization have occurred by dehydrogenation of existing cyclic rings to convert into the polyaromatic ring-like structures of graphitic planes. The powder XRD pattern confirmed the mechanism showing that sodium hydroxide was able to prevent the attack of oxygen during the pyrolysis of sugarcane bagasse, thereby preventing the formation of GO. The EDX analysis further confirmed the nonexistence of $\mathrm{GO}$ and involvement of $\mathrm{OH}$ groups of glucose monomers in the aromatization and condensation to form hexagonal polyaromatic rings, leading to the development of graphitic planes.

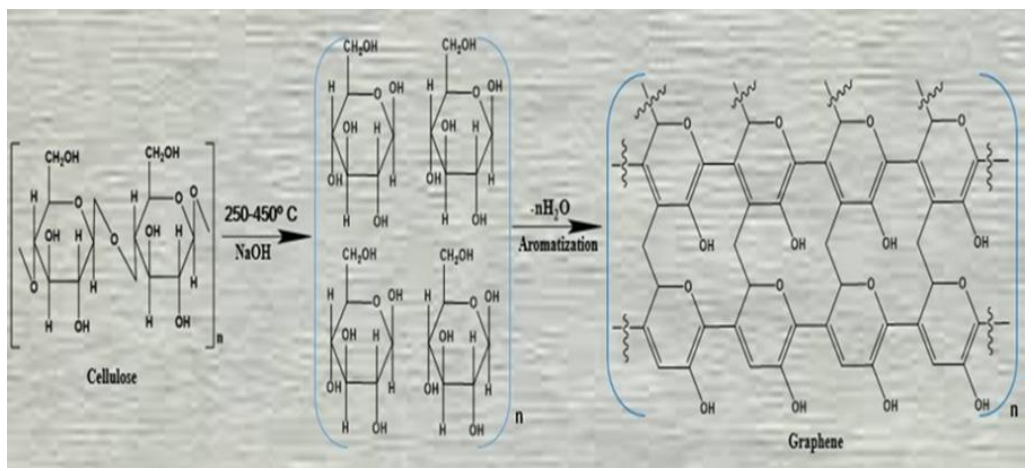

Figure 6. Mechanism of formation of graphene nanosheets at $250-350^{\circ} \mathrm{C}$. 


\section{Conclusions}

A one-step approach has been reported for the formation of graphene nanosheets by pyrolysis of sugarcane bagasse at various temperatures viz. $250^{\circ} \mathrm{C}, 350^{\circ} \mathrm{C}$, and $450^{\circ} \mathrm{C}$ in the presence of sodium hydroxide at normal atmospheric conditions, bypassing the formation of GO. The XRD pattern showed an intense peak at around $2 \theta$ of $25.6^{\circ}$ at $350^{\circ} \mathrm{C}$ temperature, which confirmed the formation of 002 planes of graphene. The SEM and TEM images further confirmed the formation of graphene nanosheets. The EDX analysis indicated that the sodium hydroxide was able to prevent the attack of oxygen during pyrolysis, thereby preventing the formation of GO. The results confirmed the chemistry of formation of graphene nanosheets in a single step, and the mechanism suggested that any hydrocarbon-rich agro-waste (cellulose$45-55 \%$, hemicellulose-20-25\%, and lignin-18-24\%) can replace the graphite powder to prepare graphene nanosheets in an environmentally, ecofriendly and energetically favorable way without high temperature and hazardous chemicals.

\section{Funding}

This research received no external funding.

\section{Acknowledgments}

We acknowledge the Central Research Facility (CRF) of NIT, Agartala for providing XRD data, FESEM from Tripura University, and NEHU, Shillong for TEM micrographs.

\section{Conflicts of Interest}

The authors declare no conflict of interest.

\section{References}

1. William, S.; Hummers, J.R.; Offeman, R.E. Preparation of graphitic oxide. J. Am. Chem. Soc. 1958, 80, 1339-1339, https://doi.org/10.1021/ja01539a017.

2. Marcano, D.C.; Kosynkin, D.V.; Berlin, J.M.; Sinitskii, A.; Sun, Z.; Slesarev, A.; Alemany, L.B.; Lu, W.; Tour, J.M. Improved synthesis of graphene oxide. ACS nano. 2010, 4, 4806-4814, https://doi.org/10.1021/nn1006368.

3. Moo, J.G.S.; Khezri, B.; Webster, R.D; Pumera, M. Graphene Oxides Prepared by Hummers', Hofmann's, and Staudenmaier's Methods: Dramatic Influences on Heavy-Metal-Ion Adsorption. ChemPhysChem 2014, 15, 2922-2929, https://doi.org/10.1002/cphc.201402279.

4. Chen, F.; Yang, J.; Bai, T.; Long, B.; Zhou, X. Facile synthesis of few-layer graphene from biomass waste and its application in lithium ion batteries. J. Electroanal.Chem. 2016, 768, 18-26, https://doi.org/10.1016/j.jelechem.2016.02.035.

5. Goswami, S.; Banerjee, P.; Datta, S.; Mukhopadhayay, A.; Das, P. Graphene oxide nanoplatelets synthesized with carbonized agro-waste biomass as green precursor and its application for the treatment of dye rich wastewater. Process Saf. Environ. 2017, 106, 163-172, https://doi.org/10.1016/j.psep.2017.01.003.

6. Shi, J.; Wang, Y.; Du, W.; Hou, Z. Synthesis of graphene encapsulated $\mathrm{Fe}_{3} \mathrm{C}$ in carbon nanotubes from biomass and its catalysis application. Carbon, 2016, 99, 330-337, https://doi.org/10.1016/j.carbon.2015.12.049.

7. Shams, S.S.; Zhang, L.S.; Hu, R.; Zhang, R; Zhu, J. Synthesis of graphene from biomass: a green chemistry approach. Mater Lett. 2015, 161, 476-479, https://doi.org/10.1016/j.matlet.2015.09.022.

8. Purkait, T.; Singh, G.; Singh, M.; Kumar, D; Dey, R.S. Large area few-layer graphene with scalable preparation from waste biomass for high-performance supercapacitor. Sci rep. 2017, 7, 1-14, https://doi.org/10.1038/s41598-017-15463-w.

9. Somanathan, T.; Prasad, K.; Ostrikov, K.K.; Saravanan, A.; Krishna, V.M. Graphene oxide synthesis from agro waste. Nanomaterials 2015, 5, 826-834, https://doi.org/10.3390/nano5020826.

10. Singh, P.; Bahadur, J.; Pal, K. One-step one chemical synthesis process of graphene from rice husk for energy storage applications. Graphene 2017, 6, 61-71, https://doi.org/10.4236/graphene.2017.63005. 
11. Silpa, P.S.; Sivmangai, N.M. One step synthesis of graphene. Inorg.Nano-Met Chem. 2019, 1-7, https://doi.org/10.1080/24701556.2019.1661470.

12. Cheng, Y.; Bi, H.; Che, X.; Li, D.; Ji, W.; Huang, F. Suppression of graphene nucleation by plasma treatment of $\mathrm{Cu}$ foil for the rapid growth of large-size single-crystal graphene. Carbon 2019, 147, 51-57, https://doi.org/10.1016/j.carbon.2019.02.025.

13. Huet, B.; Zhang, X.; Redwing, J.M.; Snyder, D.W.; Raskin, J.P. Multi-wafer batch synthesis of graphene on $\mathrm{Cu}$ films by quasi-static flow chemical vapor deposition. 2D Mater. 2019, 6.

14. Lee, I.; Bae, D.J.; Lee, W.K.; Yang, C.M.; Cho, S.W.; Nam, J.; Lee, D.Y.; Jang, A.R.; Shin, H.S.; Hwang, J.Y.; Hong, S. Rapid synthesis of graphene by chemical vapor deposition using liquefied petroleum gas as precursor. Carbon, 2019, 145, 462-469, https://doi.org/10.1016/j.carbon.2019.01.004.

15. Mansourkhani, F.; Badiei, A.; Rashidi, A.M.; Khajehmandali, S. Value-added utilization of pyrolysis heavy distillate for the synthesis of nitrogen doped graphene with chemical vapor deposition. Fuller. Nanotub. Car. N. 2019, 27, 525-530, https://doi.org/10.1080/1536383X.2019.1594198.

16. Zhao, W.; Hu, B.; Yang, Q.; Wang, Z.; Li, X.; Jin, Y.; Xi, Y.; Li, J.; Tian, W.Q. Synergetic interaction between copper and carbon impurity induces low temperature growth of highly-defective graphene for enhanced electrochemical performance. Carbon 2019, 150, 371-377, https://doi.org/10.1016/j.carbon.2019.05.046.

17. Singh, J.; Rathi, A.; Rawat, M.; Gupta, M. Graphene: from synthesis to engineering to biosensor applications. Front. Mater. Sci. 2018, 12, 1-20, https://doi.org/10.1007/s11706-018-0409-0.

18. Sultanov, F.R.; Daulbayev, C.; Bakbolat, B.; Mansurov, Z.A.; Urazgaliyeva, A.A.; Ebrahim, R.; Pei, S.S.; Huang, K.P. Microwave-enhanced chemical vapor deposition graphene nanoplatelets-derived 3D porous materials for oil/water separation. Carbon Lett 2020, 30, 81-92, https://doi.org/10.1007/s42823-019-000735 .

19. Debbarma, J.; Naik, M. J. P.; Saha, M. From agrowaste to graphene nanosheets: chemistry and synthesis. Fuller. Nanotub. Car. N. 2019, 27, 482-485, https://doi.org/10.1080/1536383X.2019.1601086.

20. Chaiyo, S.; Mehmeti, E.; Siangproh, W.; Hoang, T.L.; Nguyen, H.P.; Chailapakul, O.; Kalcher, K. Nonenzymatic electrochemical detection of glucose with a disposable paper-based sensor using a cobalt phthalocyanine-ionic liquid-graphene composite. Biosens Bioelectron. 2018, 102, 113-120, https://doi.org/10.1016/j.bios.2017.11.015.

21. Miao, F.; Wu, W.; Miao, R.; Cong, W.; Zang, Y.; Tao, B.; Graphene/nano-ZnO hybrid materials modify Nifoam for high-performance electrochemical glucose sensors. Ionics 2018, 24, 4005-4014, https://doi.org/10.1007/s11581-018-2539-X.

22. Lin, S.; Feng, W.; Miao, X.; Zhang, X.; Chen, S.; Chen, Y.; Wang, W.; Zhang, Y. A flexible and highly sensitive nonenzymatic glucose sensor based on DVD-laser scribed graphene substrate. Biosens Bioelectron. 2018, 110, 89-96, https://doi.org/10.1016/j.bios.2018.03.019.

23. Peng, Y.; Lin, D.; Gooding, J.J.; Xue, Y.; Dai, L. Flexible fiber-shaped non-enzymatic sensors with a graphene-metal heterostructure based on graphene fibres decorated with gold nanosheets. Carbon 2018, 136, 329-336, https://doi.org/10.1016/j.carbon.2018.05.004.

24. Soganci, T.; Ayranci, R.; Harputlu, E.; Ocakoglu, K.; Acet, M.; Farle, M.; Unlu, C.G.; Ak, M. An effective non-enzymatic biosensor platform based on copper nanoparticles decorated by sputtering on CVD graphene. Sensor Actuat B: Chem. 2018, 273, 1501-1507, https://doi.org/10.1016/j.snb.2018.07.064.

25. Sukhrobov, P.; Numonov, S.; Gao, S.; Mamat, X.; Wagberg, T.; Guo, Y.; Liu, L.; Hu, G. Nonenzymatic Glucose Biosensor Based on NiNPs/Nafion/Graphene Film for Direct Glucose Determination in Human Serum. Nano 2018, 13, https://doi.org/10.1142/S1793292018500753.

26. Yin, D.; Bo, X.; Liu, J.; Guo, L. A novel enzyme-free glucose and H2O2 sensor based on 3D graphene aerogels decorated with $\mathrm{Ni3N}$ nanoparticles. Anal chim acta. 2018, 1038, 11-20, https://doi.org/10.1016/j.aca.2018.06.086.

27. Chen, X.; Liu, D.; Cao, G.; Tang, Y.; Wu, C. In Situ Synthesis of a sandwich-like graphene@ ZIF-67 heterostructure for highly sensitive nonenzymatic glucose sensing in human serums. ACS appl mater inter. 2019, 11, 9374-9384, https://doi.org/10.1021/acsami.8b22478.

28. Mao, W.; He, H.; Ye, Z.; Huang, J. Three-dimensional graphene foam integrated with $\mathrm{Ni}(\mathrm{OH})_{2}$ nanosheets as a hierarchical structure for non-enzymatic glucose sensing. J Electroanal Chem 2019, 832, 275-283, https://doi.org/10.1016/j.jelechem.2018.11.016.

29. Zhu, Y.; Zhang, X.; Sun, J.; Li, M.; Lin, Y.; Kang, K.; Meng, Y.; Feng, Z.; Wang, J. A non-enzymatic amperometric glucose sensor based on the use of graphene frameworks-promoted ultrafine platinum nanoparticles. Microchim Acta. 2019, 186, 538, https://doi.org/10.1007/s00604-019-3653-9.

30. Promsuwan, K.; Kachatong, N.; Limbut, W. Simple flow injection system for non-enzymatic glucose sensing based on an electrode modified with palladium nanoparticles-graphene nanoplatelets/mullti-walled carbon nanotubes. Electrochim Acta. 2019, 320, https://doi.org/10.1016/j.electacta.2019.134621.

31. Li, M.; Li, W.; Liu, S. Hydrothermal synthesis, characterization, and $\mathrm{KOH}$ activation of carbon spheres from glucose. Carbohydr res. 2011, 346, 999-1004, https://doi.org/10.1016/j.carres.2011.03.020. 